\title{
Experiencias
}

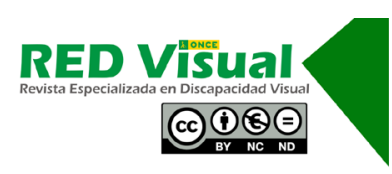

Recepción: 19-02-2021 Aceptación: 14-04-2021

\section{Actividades de lectura grupales online en época de confinamiento para alumnos con discapacidad visual: «Taller de lectura» / «Padrins lectors»}

Online group reading activities during lockdown for students with visual disability: 'Reading Workshop' / 'Padrins lectors'

\author{
L. Nadal Fiol, A. Torras Codinach, M. Sánchez Montalat, \\ À. Costa Falgàs, A. Cruañas Verdaguer, G. Iglesias Franch
}

\section{Resumen}

El confinamiento domiciliario, que empezó el 14 de marzo de 2020, nos llevó al aislamiento y, a la vez, a tener que diseñar nuevas propuestas de trabajo que permitieran dar continuidad a la atención individual y grupal de los alumnos con discapacidad visual atendidos desde el Centro de Recursos Educativos para alumnos con Discapacidad Visual-Centro de Recursos Educativos (CREDV-CRE) de la ONCE en Barcelona, subsede de Gerona. A partir de esta nueva realidad, las maestras de apoyo de dichos alumnos organizamos dos actividades de trabajo en grupo: «Padrins lectors» (destinado a alumnos con discapacidad visual expertos e iniciados en el con código de lectoescritura braille) y «Taller de lectura» (destinado a alumnos con discapacidad visual, con código de lectoescritura braille o tinta, de los ciclos medio y superior de educación primaria). Los objetivos generales para la elaboración de las propuestas de actividades grupales fueron: fomentar el vínculo entre alumnos con discapacidad visual, potenciar el interés por la lectura y el uso de las herramientas de acceso a la información. Las actividades se llevaron a cabo a través de plataformas digitales, seleccionadas en función de los requisitos de la actividad, la accesibilidad y la capacidad de acceso de los participantes y sus familias. Es importante destacar que, al inicio del confinamiento, el uso de las plataformas para realizar reuniones y, especialmente sesiones de aprendizaje en alumnos de 3 a 16 años, no estaba extendido entre la población, por lo que para el diseño y ejecución de las propuestas no dispusimos de 
experiencias previas en las que apoyarnos. La valoración que hicimos las maestras del equipo fue muy satisfactoria. No solo alcanzamos los objetivos propuestos, sino que destacamos la alta participación del alumnado, la interrelación e implicación de las familias y la posibilidad de disponer de una ventana de acceso al trabajo virtual con los alumnos que estábamos atendiendo, y así poder evaluar necesidades y promover aprendizajes.

\title{
Palabras clave
}

Confinamiento. Ceguera. Baja visión. Tiflotecnología. Competencias básicas específicas. Lectura.

\begin{abstract}
Homebound lockdown, effective beginning on 14 March 2020, brought both isolation and a need, at the Gerona Branch of ONCE's Barcelona Educational Resource Centre for Students with Visual Disability - Educational Resource Centre (CREDV CRE), to design new working models to continue to provide individualised and group support for students with visual disability. To accommodate the new situation, support teachers designed two group activities: 'Padrins lectors' geared to students with visual disability familiar with and expert users of Braille; and a 'Reading Workshop' for middle or upper primary school students with visual disability using either braille or ink format read-write code. The general objectives sought with these group activities included encouraging links among students with visual disability, heightening their interest in reading and teaching them to use tools for accessing information. Activities were undertaken on digital platforms selected on the grounds of task requirements, accessibility and openness of access to participants and their families. One important observation in this regard is that when lockdown was first instituted, neither online learning nor virtual meetings were widely extended across the population in general or 3- to 16-year-old students in particular. As a result no prior information was in place that could be used as a reference for designing and implementing proposals for action. The teaching team found the experience to be highly satisfactory. The approach was deemed to favour not only attainment of the objectives but other positive outcomes as well: high student participation rates, intensified family interrelations and involvement and the creation of a window to work virtually with students, evaluate their needs and further their learning.
\end{abstract}

\section{Key words}

Lockdown. Blindness. Low vision. Aids and appliances for visually impaired persons. Specific basic skills. Reading.

Nadal, L., Torras, A., Sánchez, M., Costa, À., Cruañas, A., e Iglesias, G. (2021). Actividades de lectura grupales online en época de confinamiento para alumnos con discapacidad visual: «Taller de lectura» / «Padrins lectors». RED Visual: Revista Especializada en Discapacidad Visual, 77, 294-304. https://doi.org/10.53094/OWBN5663. 


\section{Introducción y contexto}

Dentro del proyecto educativo del equipo del CREDV-CRE ONCE Barcelona, subsede de Gerona, queremos acentuar las actividades complementarias (AC). Dichas actividades son, prioritariamente, un espacio de relación donde se agrupan alumnos con discapacidad visual para complementar el desarrollo de las competencias básicas específicas, o sea, las capacidades que dichos alumnos deben desarrollar para adquirir eficazmente las competencias básicas escolares y así poder participar activamente en la sociedad. Dadas las características poblacionales de Gerona (número de estudiantes atendidos y dispersión geográfica) solemos contar con un grupo heterogéneo, en cuanto a edades y niveles, de unos 30 participantes (desde 3 a 16 años). Esto nos posibilita dividir al alumnado en dos grupos: niños y niñas de infantil y primaria, con una periodicidad mensual, y jóvenes de secundaria, con periodicidad trimestral. Las jornadas se desarrollan a partir de un centro de interés donde el niño o joven es el protagonista del proceso de aprendizaje con la finalidad de que sea la motivación la herramienta que permita construir y afianzar el conocimiento. Otra de las características de las AC es la organización a través de grupos flexibles. Dada la diversidad de edades, agrupamos a los niños teniendo en cuenta intereses, habilidades, necesidades específicas y/o afinidades con otros compañeros. Los grupos, modificables según la concreción de la propuesta educativa, se adaptan a las necesidades individuales de cada uno de los participantes con el fin de disponer de un entorno enriquecedor que suponga para cada uno de ellos un reto asumible. Finalmente, damos especial relevancia a la participación en las asambleas (tanto de los chicos como de las maestras de apoyo): es un espacio destinado a la escucha activa, la reflexión, la resolución de conflictos y la propuesta de cambios.

En marzo del año 2020, la situación provocada por la pandemia mundial llevó a la población española a un confinamiento domiciliario, hecho que supuso un parón, tanto en las actividades escolares como en las actividades grupales que se venían desarrollando desde el equipo de la provincia de Gerona.

Ante la incertidumbre de la duración del periodo de confinamiento, el primer objetivo que se planteó fue el acompañamiento emocional de los niños, de los jóvenes y sus familias. Al constatar que la situación se alargaría, se hizo necesario un replanteamiento de la atención individual y grupal de los alumnos con discapacidad visual. En cuanto a la atención individual (como el acceso a las actividades y contenidos curriculares que los centros planteaban o el desarrollo de habilidades específicas), el equipo de

Nadal, L., Torras, A., Sánchez, M., Costa, À., Cruañas, A., e Iglesias, G. (2021). Actividades de lectura grupales online en época de confinamiento para alumnos con discapacidad visual: «Taller de lectura» / «Padrins lectors». RED Visual: Revista Especializada en Discapacidad Visual, 77, 294-304. https://doi.org/10.53094/OWBN5663. 
maestras de Gerona acordó unos criterios de atención generales que iban sujetos a criterios como el momento educativo, el código de lectoescritura o el dominio de las TIC, los cuales debían ser adaptados a las necesidades específicas de cada alumno.

Una vez definida la metodología de intervención individual, el siguiente paso fue abordar el proyecto de actividades grupales que expondremos a continuación.

\section{Objetivos y metodología}

El objetivo principal para la elaboración de las propuestas de actividades grupales fue fomentar el vínculo entre iguales (chicos y chicas con discapacidad visual), con la certeza de que este espacio de crecimiento compartido debía ser un apoyo de gran valor en el desarrollo y ajuste emocional, especialmente en un momento de pandemia mundial y aislamiento social.

Como hemos dicho, partíamos del modelo de AC (sustentado en la flexibilidad, la motivación como eje del aprendizaje y la participación asamblearia). En la coyuntura en que nos encontrábamos, estas ideas pedagógicas cobraban mayor relevancia, pues se hacía necesario poder valorar cuáles eran los intereses y las necesidades reales de los alumnos con discapacidad visual y, a pesar de la distancia y de la complejidad de la situación, darles respuesta.

En los contactos individuales, las maestras de apoyo analizamos inquietudes y coincidimos en la necesidad manifestada por la mayoría de disponer de un punto de encuentro (que debería ser online) para dar continuidad a las relaciones construidas durante las AC.

Se era consciente de que el proyecto debía permitir impulsar la participación y la relación, respetar los diferentes ritmos de aprendizaje y fomentar el desarrollo de competencias básicas específicas. Para ello, planteamos dos talleres por grupos de edad: «Padrins lectors» y «Taller de lectura».

\section{1. «Padrins lectors»}

Una de las acepciones de la palabra padrí (padrino) es: persona que protege y acompaña a alguien en sus pretensiones y propósitos vitales. Con el grupo de «Padrins 
lectors» pretendíamos hacer llegar este valor entre compañeros de distintas edades a través de la lectura, actuando los mayores como padrinos y los pequeños como ahijados. Los primeros tendrían la oportunidad de acompañar a los menores compartiendo un cuento, y estos, a su vez, verían modelos de lectura en adolescentes mucho más próximos a ellos por edad que sus referentes adultos. Al participar también los padres de los pequeños, pensábamos que estos podrían verse también beneficiados al compartir esta experiencia con chicos y chicas con discapacidad visual en momentos evolutivos diferentes a los de sus hijos.

La actividad consistió en un encuentro virtual entre dos alumnos usuarios del código de lectura braille (un experto lector, ciclo superior de primaria - CS- o de ESO -Educación Secundaria Obligatoria-, y un iniciado en la lectura braille, último curso de educación infantil o ciclo inicial de primaria, CI) donde se leyó un cuento de manera conjunta.

En cada grupo participó un alumno de iniciación a la lectura (acompañado de sus padres), un alumno de secundaria y una maestra de apoyo del centro de recursos.

Utilizamos tres libros: El pollastre desplomat, de Chih-Yuan Chen (2010, Thule Ediciones); El regal, de Gabriela Keselman (1996, Editorial Kókinos) y La caputxeta forçuda de Albert Vitó (2013, Editorial Baula).

El proceso de desarrollo de la actividad se llevó a cabo en tres fases. En la fase previa, se encuestó a los alumnos con discapacidad visual y a las familias acerca de su interés y posibilidades de participar. Posteriormente, se procedió a la selección de los libros teniendo en cuenta el contenido y la estructura, la presencia de ilustraciones en relieve y la capacidad del Servicio de Adaptación de Recursos Didácticos y Tecnológicos (SARDT) de la ONCE de disponer de copias suficientes en un corto plazo de tiempo. Una vez seleccionados los cuentos, se facilitaron los ejemplares de manera que cada participante de educación infantil o CI dispusiera de un ejemplar de todos los libros escogidos y los de CS o de secundaria de un ejemplar de un solo libro. Se solicitó a los jóvenes la preparación de las lecturas, poniendo énfasis en la prosodia y en dar consignas verbales para entender las ilustraciones, cambios de página y contenido en general. Finalmente se concertó día, hora y canal tecnológico para realizar la conexión.

Seguidamente, se llevó a cabo la fase de desarrollo de la actividad, en la que alumnos con discapacidad visual, familias y maestras de apoyo se conectaron online 
a través del canal escogido. Tras un espacio inicial de presentación, el alumno mayor iniciaba su lectura mientras el menor podía seguirla desde su libro; el maestro intervenía, si era necesario, para ajustar el ritmo de lectura o hacer pequeñas indicaciones. El alumno pequeño participaba analizando ilustraciones o leyendo palabras o frases (según sus posibilidades). Al final de la lectura, se abría un espacio de diálogo donde se compartían experiencias, dudas o anécdotas.

Con posterioridad a los encuentros, tuvo lugar la fase de evaluación, en la que se recogieron las impresiones de las familias y alumnos con discapacidad visual para posteriormente analizarlas y proponer mejoras.

Entre las competencias básicas específicas que se pretendía trabajar, cabe destacar aquellas para las etapas de educación infantil y CI, que se iniciaban en la lectura:

- Escucha activamente.

- Saluda y se despide correctamente.

- Mantiene conversaciones propias de la edad.

- Respeta el turno de palabra.

- Reconoce y expresa sentimientos.

- Utiliza recursos específicos en función de las necesidades.

- Pide ayuda cuando la necesita.

- Transmite vivencias personales.

- Muestra interés por la lectura.

- Coloca las manos adecuadamente.

- Sigue líneas de izquierda a derecha.

- Reconoce y lee alguna letra/palabra.

Para las etapas de CS o ESO, que actuaron como expertos, las competencias básicas específicas fueron:

- Habla de temas apropiados al contexto y ajusta el registro de habla a la edad del interlocutor.

- Muestra interés en actividades colaborativas y siente necesidad de ayudar.

- Participa y domina distintas redes sociales.

- Manifiesta sus opiniones.

- Es optimista y dimensiona adecuadamente necesidades y problemas.

- Ofrece información sobre su discapacidad visual y las ayudas que necesita. 
- Muestra gusto por la lectura.

- Posee una buena fluidez lectora.

\section{2. «Taller de lectura»}

En la siguiente actividad, se creó un grupo virtual con estudiantes con discapacidad visual de ciclo medio (CM) y ciclo superior (CS) de primaria, en el cual se trabajó la lectura de un libro.

Optamos por un taller de literatura puesto que consideramos que permitía alcanzar distintos objetivos. Primeramente, se valoró que ofrecía una oportunidad para mantener el contacto con iguales y compartir con estos situaciones y vivencias personales; así mismo, ofrecía un espacio lúdico-didáctico donde se visualizaba y fomentaba el uso de las distintas herramientas de acceso a la información, y, finalmente, fomentaba el interés por la lectura.

El libro que se utilizó fue L'avi el presumpte, de Paloma Bordons (2006, Edebé). Siguiendo el modelo de «Padrins lectors», el proceso de desarrollo de la actividad se llevó a cabo, también, en tres fases. En la fase inicial, se encuestó a los alumnos con discapacidad visual sobre su interés por participar y preferencias literarias; del mismo modo, se habló con las familias para disponer de su consentimiento y apoyo. Se seleccionó el libro, teniendo en cuenta que el contenido debía ser atractivo y motivador al mismo tiempo que adecuado a las diferentes edades de los participantes, a los que se les facilitó un ejemplar accesible. Posteriormente, se concertó una reunión telemática en la que se explicó el funcionamiento y periodicidad del taller, se indicó también el capítulo o capítulos que tendrían que leer y se trabajó el uso de los comandos básicos de la aplicación usada (por ejemplo, activar y desactivar micrófono).

En la fase de desarrollo, al iniciar las sesiones, cada alumno exponía cómo había accedido (formato del libro, recursos y adaptaciones utilizadas...), si había encontrado dificultades y cómo las habían resuelto. Los jóvenes podían exponer ideas o sugerencias para los compañeros con el fin de promover un aprendizaje participativo y corresponsable. El rol de la maestra era, a pesar de la distancia física, de acompañante, orientando desde una mirada no invasiva y respetuosa.

Seguidamente, la maestra proponía preguntas para valorar el nivel de comprensión lectora (tanto a nivel literal como inferencial), promoviendo la participación equitativa 
de todos los asistentes. Para facilitar la intervención, era necesario respetar consignas de participación online (como tener los micrófonos silenciados, las cámaras activadas o levantar la mano para participar). Finalmente, se abría un turno de debate y reflexión donde maestra y jóvenes hacían hipótesis y conjeturas sobre el desenlace de los siguientes capítulos.

En la fase final de evaluación, se recogieron las impresiones de los chicos y de las maestras de apoyo para, posteriormente, analizarlas y proponer mejoras.

Entre las competencias básicas específicas que se pretendía trabajar, destacamos:

- Manifiesta una escucha activa.

- Expresa verbalmente sus opiniones de forma natural y asertiva.

- Participa, respetando los turnos de palabra, en conversaciones grupales.

- Reconoce y respeta las necesidades de los compañeros.

- Percibe la diferencia respeto al otro, con sus posibilidades y dificultades.

- Se preocupa de buscar soluciones a las dificultades que su discapacidad visual le genera.

- Participa en actividades grupales con compañeros con DV.

- Comprende la lectura y es capaz de hacer una pequeña síntesis.

\section{Valoración}

La escuela inclusiva debe garantizar que todos los niños y niñas desarrollen armónicamente todas sus capacidades y adquieran las competencias necesarias para participar activa y críticamente de la vida adulta. Los alumnos con discapacidad visual son miembros de los centros educativos de su comunidad; estos, con el apoyo de las maestras del CREDV-CRE ONCE Barcelona, deben crear los contextos de aprendizaje para que alcancen los retos educativos, entre ellos las competencias personales y sociales que les guíen en la construcción de su identidad, permitiéndoles crear vínculos afectivos y de amistad. Dicho esto, debemos destacar la importancia de la relación con iguales con discapacidad visual, que posibilita a los chicos no solamente fortalecer su autoestima, sino, especialmente, enriquecer la creación del yo, a través del reconocimiento del otro y, así, la toma de conciencia de necesidades y aptitudes.

Como es sabido, la irrupción de la covid y, seguidamente, el confinamiento y cierre de las escuelas provocaron un cambio brusco tanto en las maneras de aprender como 
en las herramientas y el acceso a los aprendizajes. Sin proceso de planificación o evaluación, los centros escolares gestionaron, a través de la comunicación digital, un nuevo sistema de relación con el objetivo de buscar alternativas que permitieran la conexión entre alumnos y docentes.

La experiencia nos dice que uno de los aspectos claves en el éxito de la inclusión educativa de los alumnos con discapacidad visual es la coordinación estrecha y permanente entre el equipo docente del centro escolar y las maestras de apoyo. Dicha coordinación permite la previsión de necesidades educativas y, por consiguiente, la anticipación de los recursos que van a facilitar el aprendizaje. Promueve, pues, una introducción coherente y significativa de las competencias específicas que harán competente al niño o adolescente en las habilidades requeridas en cada situación escolar. El cierre repentino de los centros educativos acarreó una mutación de una escuela presencial a una de digital, sin una transición que permitiera a adultos y menores un período de adaptación. En consecuencia, con los alumnos con discapacidad visual se hizo necesario incorporar nuevas estrategias adaptadas al trabajo online (tanto en la elaboración de materiales como en las aulas digitales) que no pudieron ser de implementación inmediata.

Observamos que, mientras los alumnos videntes disponían de habilidades para deducir e intuir el funcionamiento de las nuevas aplicaciones tecnológicas, los estudiantes con discapacidad visual precisaron más tiempo para ser conocedores del funcionamiento y de las estrategias para usarlas. El desequilibrio entre el alumno con discapacidad visual y sus compañeros de clase lo pudimos concretar en que disponían de menor capacidad de organización en las tareas digitales y, también, de menor flexibilidad para manejar distintas plataformas; precisaban, pues, de más tiempo y soporte por parte del adulto referente.

A pesar de que las dos propuestas que elaboramos partían de la demanda de los alumnos con discapacidad visual y sus familias, éramos desconocedoras de cómo sería su implementación, pues no disponíamos de modelos previos. Dos aspectos que nos permitieron valorar las actividades fueron la participación y las aportaciones recibidas.

En general, podemos decir que tanto la participación en número de asistentes, como en continuidad de estos fue elevada. De los chicos y chicas convocados participaron todos excepto uno, y una vez iniciadas las actividades no hubo ninguna 
baja. Es importante destacar que no pudieron participar aquellos que no disponían de medios técnicos o de apoyo familiar para seguir las sesiones online. Este punto es especialmente grave dada la situación de aislamiento en que nos encontrábamos, pues la brecha digital dejó al margen a los niños más vulnerables y, por lo tanto, con más necesidades.

A través del feedback recibido de los participantes, podemos decir que estos disfrutaron de las sesiones. Ellos destacaban que los grupos reducidos facilitaban las intervenciones y proporcionaban un espacio de mayor intimidad donde se podían sentir protagonistas, a la vez que muchos de ellos compartían la opinión de que los libros escogidos resultaban motivadores y divertidos. Por último, algunos de los chicos y chicas que formaron parte de los talleres verbalizaron que realizar estas actividades junto a compañeros con discapacidad visual les suponía menor presión que realizarlas con sus compañeros de clase. Como se ha mencionado anteriormente, el confinamiento supuso un cambio súbito en la metodología de trabajo, a la que tuvieron que adaptarse de manera brusca tanto los tutores como los alumnos, siendo imposible la anticipación. Muchos de los alumnos con discapacidad visual no disponían de las habilidades necesarias para desenvolverse en entornos digitales -tales como plataformas educativas, aulas digitales o plataformas de reuniones virtuales-. Asimismo, los maestros tutores desconocían los recursos y estrategias para facilitar el acceso y la inclusión en dichos entornos. Las dificultades que surgieron generaron sentimientos de indefensión en algunos de los estudiantes con discapacidad visual y, en determinados casos, inclusive, la percepción de un retroceso en sus progresos en autonomía. El taller de lectura les ofreció un espacio amable donde capacitarse y poder disfrutar de experiencias de éxito junto a iguales, que les empoderó.

Nos gustaría remarcar también la inestimable aportación de las familias. La situación de confinamiento facilitó que pudieran colaborar con nosotros y descubrir momentos en la educación de sus hijos que habitualmente se desarrollan en el centro educativo. Las familias destacaron que fue enriquecedor poder escuchar a otros muchachos con discapacidad visual, así como las muestras de cooperación que surgieron entre ellos.

La realización de los talleres propició a las maestras de apoyo una herramienta para poder apreciar y concretar fortalezas y debilidades en cuanto el uso y dominio de las tecnologías, y así diseñar planes de trabajo ajustado a las necesidades reales; de igual forma, nos ofreció un canal donde poder observar el momento emocional y anímico en que se encontraban. 
Finalmente, simplemente decir que, como maestras, deseamos volver a desarrollar las AC de manera presencial. Aunque con el diseño que hemos detallado logramos alcanzar los objetivos planteados, estamos convencidas de que ningún medio tecnológico puede sustituir el contacto físico que anhelamos poder recuperar pronto.

Laura Nadal Fiol. Maestra de apoyo del CREDV-CRE de la ONCE en Barcelona, subsede de Gerona. Carrer del Sol, 15; 17004 Gerona (España). Correo electrónico: Inf@once.es.

Alba Torras Codinach. Maestra de apoyo del CREDV-CRE de la ONCE en Barcelona, subsede de Gerona. Carrer del Sol, 15; 17004 Gerona (España). Correo electrónico: atorra27@xtec.cat.

Mariona Sánchez Montalat. Maestra de apoyo del CREDV-CRE de la ONCE en Barcelona, subsede de Gerona. Carrer del Sol, 15; 17004 Gerona (España).Correo electrónico: msmo@once.es.

Ànnia Costa Falgàs. Maestra de apoyo del CREDV-CRE de la ONCE en Barcelona, subsede de Gerona. Carrer del Sol, 15; 17004 Gerona (España). Correo electrónico: acosta43@xtec.cat.

Alexandra Cruañas Verdaguer. Maestra de apoyo del CREDV-CRE de la ONCE en Barcelona, subsede de Gerona. Carrer del Sol, 15; 17004 Gerona (España).Correo electrónico: acruanas@xtec.cat.

Gemma Iglesias Franch. Maestra de apoyo del CREDV-CRE de la ONCE en Barcelona, subsede de Gerona. Carrer del Sol, 15; 17004 Gerona (España). Correo electrónico: giglesi6@xtec.cat. 